\title{
Alpha-Adducin Gly460Trp Polymorphism and Clinical Efficacy of Indapamide in Uzbek Hypertensive Patients
}

\author{
Guzal J. Abdullaeva*, PhD; Gulnoz A. Khamidullaeva, PhD, ScD \\ The Republican Specialized Center of Cardiology \\ Tashkent, Uzbekistan
}

\begin{abstract}
The purpose of the present study was to evaluate pharmacogenetic aspects of the antihypertensive, cardioprotective and vasoprotective efficacy of Indapamide in association with the ADD1 Gly460Trp polymorphism in Uzbek hypertensive patients.

Materials and Methods: The study included 37 ethnic Uzbek patients (mean age of 47.14 \pm 9.54 years) with untreated Hypertension (HT) of Grade 1 and 2 (ESH/ESC, 2013) and average HT duration of 5.7 \pm 4.33 years. All patients underwent clinical examination, echocardiography, Doppler sonography study, assessment of flow-mediated dilation (FMD) of the brachial artery and microalbuminuria (MAU) in daily urine. Genomic DNA was extracted from peripheral blood using the Diatom ${ }^{\mathrm{TM}}$ DNA Prep $200 \mathrm{Kit}$ according to the manufacturer's protocol. The PCR-RFLP technique with visualization was performed to determine the ADD1 Gly460Trp polymorphism. Indapamide was prescribed as monotherapy for 12 weeks with an initial dose of $2.5 \mathrm{mg}$.

Results: A 12-week monotherapy with Indapamide in a daily dose of $2.5 \mathrm{mg}$ showed the high antihypertensive efficacy of this drug expressing a reliable decrease in absolute values of SBP and DBP independently as carrying of the ADD1 Gly460Trp polymorphism allele. Positive changes in LVM during therapy in patients of both allele groups were accompanied by an improvement in LV diastolic function with significant positive dynamics of IRP. The target normalization of FMD was achieved only in the presence of Gly-allele, as well as a significant decrease in IMT and MAU during treatment.

Conclusion: The results of our study showed high antihypertensive and cardioprotective efficacy of Indapamide treatment independently on carrying of the ADD1 Gly460Trp polymorphism. At the same time, we revealed certain advantages in the vasoprotective efficacy of Indapamide treatment in Uzbek hypertensive patients who are carriers of Gly-allele of the ADD1 Gly460Trp polymorphism. (Int J Biomed. 2015;5(3):132-136.)
\end{abstract}

Keywords: hypertension; Indapamide; alpha-adducin Gly460Trp polymorphism.

Abbreviations: BP, blood pressure; LVEDD, left ventricular end-diastolic dimension; LVESD, left ventricular end-systolic dimension; IVST, interventricular septal thickness; PWT, posterior wall thickness; LVMI, left ventricular mass index; PE, peak early filling velocity; PA, peak atrial filling velocity; IRP, isovolumic relaxation phase; IMT, intima-media thickness; FMD, flow-mediated dilation; MAU, microalbuminuria.

\section{Introduction}

Hypertension(HT) is a main risk factor for cardiovascular mortality. Currently, HT control is the most significant way to reduce cardiovascular risk [1-5]. However, different approaches exist in the choice of antihypertensive drugs. Individual response to antihypertensive therapy, as well as the spectrum of unwanted side effects, promotes poor adherence to treatment. The variability in drug response is of great

*Corresponding author: Guzal J. Abdullaeva, PhD. The Republican Specialized Center of Cardiology Tashkent, Uzbekistan. E-mail: guzal-abdullaeva@bk.ru interest for pharmacogenetic studies. The HT treatment should start with thiazide diuretics in accordance with guidelines for the management of HT (ESC/ESH, 2013). Indapamide has a special place among diuretics due to its high efficacy, a good tolerability, and metabolic neutrality [6-9]. About $60 \%$ to $70 \%$ percent of indapamide is eliminated through the kidneys. Sodium retention remains the cornerstone of the pathogenesis and the treatment of HT, and thiazide diuretics remain as drugs of choice in treatment of hypertensive patients in the general population [1]. Nevertheless, not all hypertensive patients are salt-sensitive, and patient response to sodium retention and diuretics is variable [10-11].

Adducin is a heterodimeric cytoskeleton protein 
comprising either alpha/beta or alpha/gamma heterodimers. Its subunits are encoded by 3 related genes (ADD1, ADD2, and ADD3, which show alternative spliced variants) located on different chromosomes [12]. The $\alpha$-subunit is known to increase renal sodium reabsorption and may be involved in the pathophysiology of essential hypertension. The human ADD1 gene is located on chromosome 4p16.3 and comprises 16 exons [13]. ADD1 gene is considered as a major candidate gene for $\mathrm{AH}$. One of the well-studied polymorphisms in the ADD1 gene is a substitution of Gly for Trp at amino acid residue 460 (G460W, rs4961). In clinical studies, some have reproduced the supportive association between Gly460Trp polymorphism and AH or BP level [14-17], whereas others were unable to replicate these findings [18-22].

The ADD1 Gly460Trp polymorphism, C825T polymorphism of the GNB3 gene, and $\beta$-T594M polymorphism of the epithelial sodium channel gene are genetic markers associated with expression of albumens that are connected with renal sodium transport. Of these polymorphisms, the ADD1 Gly460Trp polymorphism has the biggest influence on the relationship between the efficacy of diuretics treatment and sodium sensitivity.

The aim of this study was to evaluate pharmacogenetic aspects of the antihypertensive, cardioprotective and vasoprotective efficacy of Indapamide (Indap, Pro.Med.CS, Czech Republic) in association with the ADD1 Gly460Trp polymorphism in Uzbek hypertensive patients.

\section{Materials and Methods}

The study included 37 ethnic Uzbek patients (mean age of $47.14 \pm 9.54$ years) with untreated HT of Grade 1 and 2 (ESH/ESC, 2013) and average HT duration of $5.7 \pm 4.33$ years. The study was approved by the Republican Specialized Center of Cardiology Ethics Committee. Written informed consent was obtained from each patient. The diagnosis of HT was based on $2013 \mathrm{ESH} / \mathrm{ESC}$ Guidelines for the management of arterial hypertension [3]. Exclusion criteria were symptomatic hypertension, clinical evidence of cerebrovascular or coronary heart diseases, cardiac arrhythmia, heart failure, renal impairment, diabetes mellitus, metabolic and other background diseases, alcohol intake greater than $30 \mathrm{~g}$ of pure ethanol per day, and smoking.

Echocardiography and Doppler sonography study carried out on the unit «EnVisorC $\AA$ » by standard methods using the recommendations of the American Society of echocardiography (Sahn DJ, Demaria A, 1987) The following parameters were measured and calculated: IVST, PWT, LVEDD, LVESD, EF, LVEVD, LVESV, and LVM (LVM was calculated using the formula R. Devereux (1977). LVM was indexed to body surface area (LVMI). Left ventricular hypertrophy (LVH) was defined as LVMI of $>95 \mathrm{~g} / \mathrm{m}^{2}$ (for women) and $>115 \mathrm{~g} / \mathrm{m}^{2}$ (for men).

The ratio of peak early filling velocity to peak atrial filling velocity (PE/PA) was calculated. The isovolumic relaxation phase (IRP) was also measured.

Carotid artery intima-media thickness (IMT) was measured by a $7.5 \mathrm{MHz}$ high-resolution ultrasound
(EnVisorC®). IMT was defined as maximum thickness of IMT at the region of interest detected in both left and right carotid artery including common carotid artery.

Assessment of flow-mediated dilation (FMD) of the brachial artery has been used as a method of determining endothelial function. The diameter of the brachial artery was measured from two dimensional ultrasound images, with the 7.5 MHz linear array transducer and standard EnVisorC $\mathbb{R}$ system. In each study, scans were taken at rest and during reactive hyperemia. FMD was estimated as the percent change in the diameter relative to the baseline diameter at rest. Level of $\mathrm{FMD} \geq 10 \%$ was taken as the norm threshold based on the Celemajer works [24].

The degree of microalbuminuria (MAU) in daily urine was determined with enzymatic method using biochemical analyzer ("Daytona TM", «Rendox», UK), which allows evaluate the MAU within $20-100 \mathrm{mg} / \mathrm{l}$ and above.

Genomic DNA was extracted from peripheral blood using the Diatom ${ }^{\mathrm{TM}}$ DNA Prep 200 Kit according to the manufacturer's protocol. The polymerase chain reactionrestriction fragment length polymorphism (PCR-RFLP) technique with visualization was performed according to previously described methodologies to determine the ADD1 Gly460Trp polymorphism [23].

Indapamide was prescribed as monotherapy for 12 weeks with an initial dose of $2.5 \mathrm{mg}$.

Statistical analysis was performed using the statistical software «Statistica» (v6.0, StatSoft, USA). Baseline characteristics were summarized as frequencies and percentages for categorical variables and as mean $\pm \mathrm{SD}$ for continuous variables. Student's unpaired and paired t-tests were used to compare two groups for data with normal distribution. Deviation from Hardy-Weinberg equilibrium and differences in allele distributions between the two groups were assessed by $\chi^{2}$ - test with 1 degree of freedom (df). Twotailed $P$ values $<0.05$ were considered statistically significant.

\section{Results}

Distribution of alleles of ADD1 Gly460Trp polymorphism in patients was similar to our early study [25]. In particular, among hypertensive patients $(n=160)$ Gly-allele was revealed in $76.9 \%$ of cases, and Trp-allele in $23.1 \%$ of cases $\left(\chi^{2}=182.75, \mathrm{df}=1, P=0.000\right)$; herewith, in normotensive subjects $(\mathrm{n}=58)$ the Gly and Trp allele frequency was $71.6 \%$ and $28.4 \%$, respectively $\left(\chi^{2}=41.39, \mathrm{df}=1, P=0.000\right.$ [25]). The significant accumulation of Gly-allele of the ADD1 Gly460Trp polymorphism was shown. The allele frequency did not differ significantly between the hypertensive and normotensive subjects. Due to the small number of patients subjected to 12-week indapamide treatment, only allele analysis of antihypertensive and antiremodeling efficacy was performed. Patients were divided into two groups: Group 1 - Gly-allele carriers $(\mathrm{n}=53)$ and Group 2 - Trp-allele carriers $(\mathrm{n}=21)$, $71.6 \%$ and $28.4 \%$, respectively $\left(\chi^{2}=29.973, \mathrm{df}=1, P=0.000\right)$. According to the initial level of BP and the initial parameters of LVH and ED, the Gly and Trp allele carriers of ADD1 Gly460Trp polymorphism were not different from each other. 


\section{Estimation of antihypertensive efficacy of Indapamide}

A 12-week monotherapy with indapamide in a daily dose of $2.5 \mathrm{mg}$ showed the high antihypertensive efficacy of this drug expressing a reliable decrease in absolute values of SBP and DBP independently as carrying of the ADD1 Gly460Trp polymorphism allele (Table 1). In two study groups, the high level of BP reduction estimated by the degree of reduction in mean blood pressure $>10 \%(-18.2 \pm 5.9 \%$ for Group 1 and $-16.4 \pm 4.7 \%$ for Group 2 ) was noted. Positive antihypertensive efficacy was noted in $92.5 \%$ of cases for Group 1 and $90.5 \%$ cases for Group 2. Achievement of the target level of SBP was noted in $83 \%$ of cases for Group 1 and $95.2 \%$ of cases for Group $2\left(\chi^{2}=1.382, \mathrm{df}=1, P=0.240\right)$. Achievement of the target level of DBP was noted in $83 \%$ of cases for Group 1 and $90.5 \%$ of cases for Group $2\left(\chi^{2}=0.401, \mathrm{df}=1, P=0.526\right)$. Simultaneous achievement of target levels of SBP and DBP was noted in $75.5 \%$ of cases in Group 1 and $85.7 \%$ of cases in Group 2. It is possible to note a certain tendency towards a better antihypertensive response in patients with Trp-allele of the ADD1 Gly460Trp polymorphism.

Table 1.

Antihypertensive efficacy of 12-week monotherapy with Indapamide in association with the ADD1 Gly460Trp polymorphism

\begin{tabular}{|l|c|c|}
\hline \multicolumn{1}{|c|}{ Variable } & Group 1 & Group 2 \\
\hline SBP, mmHg & $\frac{151.79 \pm 11.6}{124.62 \pm 10.04^{*}}$ & $\underline{149.3 \pm 7.46}$ \\
\hline DBP, mmHg & $\frac{96.6 \pm 8.25}{78.5 \pm 7.94^{*}}$ & $\underline{94.29 \pm 6.57}$ \\
\hline Mean BP, mmHg & $\underline{115.0 \pm 8.22}$ & $\underline{112.62 \pm 6.15}$ \\
\hline SBP, $\Delta \%$ & $93.87 \pm 7.63^{*}$ & $94.05 \pm 5.76^{*}$ \\
\hline DBP, $\Delta \%$ & $-17.8 \pm 5.4$ & $-15.6 \pm 4.3$ \\
\hline Mean BP, $\Delta \%$ & $-18.4 \pm 8.8$ & $-16.96 \pm 7.8$ \\
\hline $\begin{array}{l}\text { Achievement of target levels of } \\
\text { SBP, } \%\end{array}$ & $-18.2 \pm 5.9$ & $-16.4 \pm 4.7$ \\
DBP, $\%$ & $83 \%$ & $95.2 \%$ \\
\hline $\begin{array}{l}\text { Achievement of target levels of } \\
\text { SBP and DBP, } \%\end{array}$ & $75.5 \%$ & $80.5 \%$ \\
\hline
\end{tabular}

Note: *- $P<0.001$ between before and after treatment; numeratorbefore, denominator - after treatment.

\section{Estimation of cardioprotective efficacy of Indapamide}

Our analysis revealed no certain advantages of antiremodeling therapy depending on the allelic carrier (Table 2). A significant decrease in IVST and PWT was shown in two study groups. We revealed no differences in degree of IVST and PWT reduction: $-5.37 \pm 6.93 \%$ vs. $-6.05 \pm 7.98 \%(P>0.05)$ for IVST in Groups 1 and 2, respectively; $-7.79 \pm 7.91 \%$ vs. $-5.06 \pm 8.78 \%(P>0.05)$ for PWT in Groups 1 and 2, respectively. The revealed dynamics of LV wall thickness were accompanied by a significant regression of LVM (Table
2); the degree of LVMI reduction was $-13.4 \pm 9.5 \%$ in Group 1 and $-10.9 \pm 9.4 \%$ in Group $2(P>0.05)$. A significant reduction of LVM in carriers of Gly-allele during indapamide therapy was associated with a reduction in the degree of the concentric LVH. It should be noted that only Group 1 patients showed a significant increase in the EDV/LVM ratio: $0.63 \pm 0.10 \mathrm{ml} / \mathrm{g}$ before treatment and $0.69 \pm 0.10 \mathrm{ml} / \mathrm{g}$ after treatment $(P<0.02)$ compared to Group 2 patients: $0.63 \pm 0.13 \mathrm{ml} / \mathrm{g}$ before treatment and $0.68 \pm 0.14 \mathrm{ml} / \mathrm{g}$ after treatment $(P>0.05)$. Such positive changes in LVM during therapy in patients of both groups were accompanied by an improvement in LV diastolic function with significant positive dynamics of IRP.

Table 2.

Cardioprotective efficacy of 12-week monotherapy with Indapamide in association with the ADD1 Gly460Trp polymorphism

\begin{tabular}{|c|c|c|}
\hline Variable & Group 1 & Group 2 \\
\hline IVST, cm & $\frac{1.05 \pm 0.13}{0.96 \pm 0.12 * * *}$ & $\frac{1.06 \pm 0.15}{1.01 \pm 0.15^{* *}}$ \\
\hline PWT, cm & $\frac{0.88 \pm 0.14}{0.83 \pm 0.12 *}$ & $\frac{0.91 \pm 0.22}{0.85 \pm 0.22 * *}$ \\
\hline EDV/LVM, ml/g & $\frac{0.63 \pm 0.10}{0.69 \pm 0.10 * *}$ & $\frac{0.63 \pm 0.13}{0.68 \pm 0.14}$ \\
\hline $\mathrm{EDD}, \mathrm{cm}$ & $\frac{5.55 \pm 0.49}{5.41 \pm 0.50}$ & $\frac{5.66 \pm 0.36}{5.54 \pm 0.34}$ \\
\hline $\mathrm{ESD}, \mathrm{cm}$ & $\frac{3.37 \pm 0.34}{3.29 \pm 0.36}$ & $\frac{3.44 \pm 0.25}{3.36 \pm 0.25}$ \\
\hline $\mathrm{EF}, \%$ & $\frac{69.20 \pm 3.29}{68.86 \pm 3.84}$ & $\frac{68.94 \pm 3.43}{69.20 \pm 3.75}$ \\
\hline $\mathrm{PE} / \mathrm{PA}$ & $\frac{1.04 \pm 0.31}{1.03 \pm 0.24}$ & $\frac{1.06 \pm 0.30}{1.10 \pm 0.27}$ \\
\hline IRP, sec & $\frac{0.100 \pm 0.02}{0.092 \pm 0.02 *}$ & $\frac{0.100 \pm 0.01}{0.085 \pm 0.03^{*}}$ \\
\hline LVM, g & $\frac{247.47 \pm 62.70}{213.77 \pm 53.20 * *}$ & $\frac{263.24 \pm 67.43}{235.2 \pm 72.17 * * *}$ \\
\hline LVMI, g/m² & $\frac{124.77 \pm 26.01}{107.70 \pm 22.49 * * *}$ & $\frac{132.19 \pm 29.22}{117.8 \pm 30.57^{* * *}}$ \\
\hline IVST, $\Delta \%$ & $-5.37 \pm 6.93$ & $-6.05 \pm 7.98$ \\
\hline $\mathrm{PWT}, \Delta \%$ & $-7.79 \pm 7.91$ & $-5.06 \pm 8.78$ \\
\hline LVMI, $\Delta \%$ & $-13.4 \pm 9.5$ & $-10.9 \pm 9.4$ \\
\hline
\end{tabular}

Note. * $P<0.05, * *_{-} P<0.02, * * *_{-} P<0.001$-- between before and after treatment; numerator - before, denominator - after treatment.

\section{Estimation of vasoprotective efficacv of Indapamide}

The analysis of the vasoprotective efficacy of Indapamide (Table 3) by the dynamics of FMD, IMT, and MAU testified to the drug's benefits in Group 1 patients. In particular, on the background of positive dynamics of FMD in patients of both groups, the target normalization of FMD was achieved only in the presence of Gly-allele (10.60 $\pm 4.19 \%)$. Only in Group 1 patients did we find a significant decrease in IMT and MAU during treatment (Table 3). 
Table 3.

Vasoprotective efficacy of 12-week monotherapy with Indapamide in association with the ADD1 Gly460Trp polymorphism

\begin{tabular}{|l|c|c|}
\hline \multicolumn{1}{|c|}{ Variable } & Group 1 & Group 3 \\
\hline $\mathrm{D}, \Delta \%$ & $\underline{9.47 \pm 3.91}$ & $\underline{8.85 \pm 4.14}$ \\
\hline MAU, mg/l & $\underline{10.60 \pm 4.19}$ & $9.73 \pm 3.61$ \\
\hline IMT, mm & $\underline{16.11 \pm 18.73}$ & $\underline{13.83 \pm 8.05}$ \\
\hline MAU, $\Delta \%$ & $0.86 \pm 0.94^{*} * 0.15 *$ & $10.05 \pm 10.12$ \\
\hline
\end{tabular}

Note. ${ }_{-} P<0.05, *_{-} P<0.001$-- between before and after treatment; numerator - before, denominator - after treatment.

\section{Discussion}

In the current study, we present the results of the pharmacogenetic features of Indapamide in association with the ADD1 Gly460Trp polymorphism in Uzbek hypertensive patients.

D. Cusi et al. [14] first identified the Gly460Trp polymorphism and showed the increased response to a decrease in serum sodium and diuretic therapy in hypertensive patients who were the 460Trp-allele carriers [14]. Subsequent studies have found an increased pressure response to salt load in carriers of the $460 \mathrm{Trp}$-allele suffering from HT compared to Gly/Gly-homozygous HT individuals [26]. In two additional studies of the same research group, the significantly greater antihypertensive response to hydrochlorothiazide was found among the 460Trp-allele carriers [27,28]. In the study of Grant and others, a significantly greater decrease in SBP was revealed in HT patients who were homozygous for the 460Trp-allele than in heterozygous or homozygous individuals for the Gly460-allele $(-25 \pm 4 \mathrm{mmHg}$ vs. $-12 \pm 2 \mathrm{mmHg}$ and $-14 \pm 1 \mathrm{mmHg}$, respectively, $P<0.05)$. This peculiarity might be caused by environmental conditions. In our study, the antihypertensive efficacy of indapamide was equally high for Gly-allele carriers and Trp-allele carriers. However, a tendency towards a better antihypertensive response with achievement of target levels of SBP and DBP was revealed in patients with Trp-allele of the ADD1 Gly460Trp polymorphism, which is in agreement with other studies. In addition, many patients, carriers of the Trp/Trp- genotype, were classified as carriers of a "low-renin" form of HT (66.7 \%) compared with other patients $(23.8 \%)[29,30]$. In contrast to the well-designed study on the ADD1 Gly460Trp polymorphism, some other studies in other populations have shown insufficient results to confirm the same correlation between ADD1 polymorphism and sodium sensitivity [29,31] or a response to diuretic therapy [32], which causes great interest in continuing the investigations. A multiple choice of antihypertensive drugs is widely available. However, a high individual variability to the antihypertensive therapy is still responsible for only a modest reduction of the $\mathrm{CV}$ risk and unsatisfactory control of
BP levels. The success of future hypertension treatment will depend upon understanding the genetic molecular mechanisms operating in subsets of patients, and the ability of new drugs to purposefully correct such alterations. For example, the study [33] of three genes (ADD1, NEDD4L, and WNK1) related to renal sodium handling and blood pressure regulation showed impressive results. In particular, SBP decreased by $11.2 \mathrm{mmHg}$ in patients with one mutated allele (ADD1 Trp), and by 15.2 mmHg in patients with two mutated alleles (ADD1 Trp and WNK1 GG). Carriers of three mutated alleles (ADD1 Trp, WNK1 GG, and NEDD4L G) were the diuretic "sensitive" patients: SAD decreased by $23.2 \mathrm{mmHg}$. Finally, on the other hand, a decrease in SAD was only $3.4 \mathrm{mmHg}$ in patients carrying the wild type alleles (WNK1 AA, NEDD4L AA) on the background of the ADD1 Trp allele. Thus, the authors found the ADD1 Trp-allele to be permissive for the effects of variants of the other genes.

In conclusion, the results of our study showed high antihypertensive and cardioprotective efficacy of Indapamide treatment independently on carrying of the ADD1 Gly460Trp polymorphism. At the same time, we revealed certain advantages in the vasoprotective efficacy of Indapamide treatment in Uzbek hypertensive patients who are carriers of Gly-allele of the ADD1 Gly460Trp polymorphism.

\section{Competing interests}

The authors declare that they have no competing interests.

\section{References}

1. Chobanian AV, Bakris GL, Black HR, Cushman WC, Green LA, Izzo JL Jr, et al. Seventh report of the Joint National Committee on prevention, detection, evaluation and treatment of high blood pressure. J Hypertens. 2003;42(6):1206-52.

2. Whitworth JA; World Health Organization, International Society of Hypertension Writing Group. 2003 World Health Organization (WHO)/International Society of Hypertension (ISH) statement on management of hypertension. J Hypertens. 2003;21(11):1983-92.

3. Mancia G, Fagard R, Narkiewicz K, Redon J, Zanchetti A, Böhm M, et al. $2013 \mathrm{ESH} / \mathrm{ESC}$ guidelines for the management of arterial hypertension: the Task Force for the Management of Arterial Hypertension of the European Society of Hypertension (ESH) and of the European Society of Cardiology (ESC). Eur Heart J. 2013;34(28):2159-219.

4. The diagnostics and treatment of arterial hypertension. Recommendations of Russian Medical Society of Arterial Hypertension and All-Russian Society of Cardiology. Kardiovas Terap Prof . 2008;7(6 Suppl 2). [in Russian].

5. Mancia G, Laurent S, Agabiti-Rosei E, Ambrosioni E, Burnier M, Caulfield MJ, et al. Reappraisal of European guidelines on hypertension management: a European Society of Hypertension Task Force document. J Hypertens. 2009; 27(11):2121-58.

6. Preobrajenskiy DV, Sidorencko BA. Treatment of arterial hypertension. Part I. Moscow; 1999 [Monograph in Russian]. 7. Sidorenko BA, Preobrajenskiy DV. Quick manual for the treatment of essential hypertension. Moscow; 1997 [Manual in Russian]. 
8. Kaplan NM. Diuretics: cornerstone of antihypertensive therapy. Am J Cardiol. 1996;77(6):3B-5B.

9. Sidorenko BA, Preobrajenskiy DV. Diagnostics and treatment of arterial hypertension. Part II. Москва; 2000. [Monograph in Russian].

10. Materson BJ, Reda DJ, Cushman WC, Massie BM, Freis ED, Kochar MS, et al. Single-drug therapy for hypertension in men. A comparison of six antihypertensive agents with placebo. The Department of Veterans Affairs Cooperative Study Group on Antihypertensive Agents. N Engl J Med. 1993(13);328:914-21.

11. Luft FC, Weinberger MH. Heterogeneous responses to changes in dietary salt intake: the salt-sensitivity paradigm. Am J Clin Nutr. 1997;65(2 Suppl): 612S-617S.

12. Matsuoka Y, Li X, Bennett V. Adducin: structure, function and regulation. Cell Mol Life Sci. 2000;57(6):884-95.

13. Lin B, Nasir J, Mc Donald H, Graham R, Rommens JM, Goldberg YP, et al. Genomic organization of the human alphaadducin gene and its alternately spliced isoforms. Genomics. 1995;25(1):93-9.

14. Cusi D, Barlassina C, Azzani T, Casari G, Citterio L, Devoto M, et al. Polymorphisms of alpha-adducin and salt sensitivity in patients with essential hypertension. Lancet. 1997;349(9062):1353-57.

15. Tamaki S, Iwai N, Tsujita Y, Nakamura Y, Kinoshita M. Polymorphism of alpha-adducin in Japanese patients with essential hypertension. Hypertens Res. 1998;21(1):29-32.

16. Barlassina C, Norton GR, Samani NJ, Woodwiss AJ, Candy GC, Radevski I, et al. Alpha-adducin polymorphism in hypertensives of South African ancestry. Am J Hypertens. 2000;13(6 Pt 1):719-23.

17. Ju Z, Zhang H, Sun K, Song Y, Lu H, Hui R, Huang $\mathrm{X}$. Alpha-adducin gene polymorphism is associated with essential hypertension in Chinese: a case-control and familybased study. J Hypertens. 2003;21(10):1861-8.

18. Kato N, Sugiyama T, Nabika T, Morita H, Kurihara H, Yazaki Y, et al. Lack of association between the alpha-adducin locus and essential hypertension in the Japanese population. Hypertension. 1998;31(3):730-3.

19. Ishikawa K, Katsuya T, Sato N, Nakata Y, Takami S, Takiuchi S, et al. No association between alpha-adducin 460 polymorphism and essential hypertension in a Japanese population. Am J Hypertens. 1998;11(4 Pt 1):502-6.

20. Alam S, Liyou N, Davis D, Tresillian M, Johnson AG. The 460Trp polymorphism of the human alpha-adducin gene is not associated with isolated systolic hypertension in elderly Australian Caucasians. J Hum Hypertens. 2000;14(3):199-203. 21. Larson N, Hutchinson R, Boerwinkle E. Lack of association of 3 functional gene variants with hypertension in African Americans. Hypertension. 2000;35(6):1297-300.
22. He X, Zhu DL, Chu SL, Jin L, Xiong MM, Wang GL, et al. Alpha-adducin gene and essential hypertension in China. Clin Exp Hypertens. 2001;23(7):579-89.

23. Castejon AM, Alfieri AB, Hoffmann IS, Rathinavelu A, Cubeddu LX. Alpha adducin polymorphism, salt sensitivity, nitric oxide excretion, and cardiovascular risk factors in normotensive Hispanics. Am J Hypertens. 2003;16(12):1018-24. 24. Celermajer DS, Sorensen KE, Gooch VM, Spiegehalter DJ, Miller OI, Sullivan ID, et al. Non-invasive detection of endothelial dysfunction in children and adults at risk of atherosclerosis. Lancet. 1992;340(8828):1111-5.

25. Eliseyeva MR, Khamidullaeva GA, Srojidinova NZ, Abdullaeva GJ, Kurbanova DR, Karimova BSh, et al. Molecular genetics aspects of essential hypertension. Tashkent: SHARK; 2009. [Monograph in Russian].

26. Barlassina C, Schork NJ, Manunta P, Citterio L, Sciarrone M, Lanella G, et al. Synergistic effect of alpha-adducin and ACE genes causes blood pressure changes with body sodium and volume expansion. Kidney Int. 2000;57(3):1083-90.

27. Sciarrone MT, Stella P, Barlassina C, Manunta P, Lanzani $\mathrm{C}$, Bianchi $\mathrm{G}$, et al. ACE and $\alpha$-adducin polymorphism as markers of individual response to diuretic therapy. Hypertension. 2003; 41(3):398-403.

28. Glorioso N, Manunta P, Filigheddu F, Troffa C, Stella $\mathrm{P}$, Barlassina $\mathrm{C}$, et al. The role of $\alpha$-adducin polymorphism in blood pressure and sodium handling regulation may not be excluded by a negative association study. Hypertension. 1999;34(4 Pt 1):649-54.

29. Grant FD, Romero JR, Jeunemaitre X, Hunt SC, Hopkins PN, Hollenberg NH, et al. Low-renin hypertension, altered sodium homeostasis, and an alpha-adducin polymorphism. Hypertension. 2002;39(2):191-6.

30. Winnicki M, Somers VK, Accurso V, Hoffmann M, Pawlowski R, Frigo G, et al. Alpha-Adducin Gly460Trp polymorphism, left ventricular mass and plasma renin activity. J Hypertens. 2002;20(9):1771-7.

31. Ciechanowicz A, Widecka K, Drozd R, Adler G, Cyrylowski L, Czekalski S. Lack of association between Gly460Trp polymorphism of alpha-adducin gene and salt sensitivity of blood pressure in Polish hypertensives. Kidney Blood Press Res. 2001;24(3):201-6.

32. Turner ST, Chapman AB, Schwartz GL, Boerwinkle E. Effects of endothelial nitric oxide synthase, a-adducin, and other candidate gene polymorphisms on blood pressure response to hydrochlorothiazide. Am J Hypertens. 2003; 16(10):834-9.

33. Manunta P, Lavery G, Lanzani C, Braund PS, Simonini $\mathrm{M}$, Bodycote $\mathrm{C}$ et al. Physiological interaction between alphaadducin and WNK1-NEDD4L pathways on sodium related blood pressure regulation. Hypertension. 2008;52(2):366-72. 